\title{
Structural and dynamical properties of a quasi-one-dimensional classical binary system
}

\author{
W. P. Ferreira, ${ }^{1, *}$ J. C. N. Carvalho, ${ }^{1}$ P. W. S. Oliveira, ${ }^{1}$ G. A. Farias,${ }^{1}$ and F. M. Peeters ${ }^{2, \dagger}$ \\ ${ }^{1}$ Departamento de Física, Universidade Federal do Ceará, Caixa Postal 6030, Campus do Pici, \\ 60455-760 Fortaleza, Ceará, Brazil \\ ${ }^{2}$ Department of Physics, University of Antwerp, Groenenborgerlaan 171, B-2020 Antwerpen, Belgium \\ (Received 22 October 2007; revised manuscript received 21 December 2007; published 31 January 2008)
}

\begin{abstract}
The ground state configurations and the "normal" mode spectra of a quasi-one-dimensional binary system of charged particles interacting through a screened Coulomb potential are presented. The minimum energy configurations were obtained analytically and independently through molecular dynamic simulations. A rich variety of ordered structures was found as a function of the screening parameter, the particle density, and the ratio between the charges of the distinct types of particles. Continuous and discontinuous structural transitions as well as an unexpected symmetry breaking in the charge distribution are observed when the density of the system is changed. For nearly equal charges, we found a disordered phase where a mixing of the two types of particles occurs. The phonon dispersion curves were calculated within the harmonic approximation for the oneand two-chain structures.
\end{abstract}

DOI: 10.1103/PhysRevB.77.014112 PACS number(s): 64.60.Cn, 82.70.Dd, 63.20.-e, 83.10.-y

\section{INTRODUCTION}

Advances in experimental and numerical techniques allowed a considerable improvement of the possibilities to create and study new crystalline structures which will open a vast field of scientific and technological applications. Recently, a variety of novel three-dimensional (3D) binary superlattices obtained through the combination of semiconducting, metallic, and magnetic colloidal nanoparticles was observed experimentally. ${ }^{1,2}$ The self-organization in a twodimensional (2D) binary colloidal system resulted in interesting mixed configurations with partial clustering. ${ }^{3}$ The use of colloidal particles is technically interesting since it allows a real time and spatial direct observation of their position, which is a great advantage as compared to atoms or molecules, as shown recently in an interesting experimental study concerning defect induced melting. ${ }^{4}$

Systems of particles moving in space of a reduced dimensionality or submitted to an external confinement exhibit different behavior from their free-of-border counterparts. It has been shown previously that the presence of constraints generates new features which are in some cases unexpected, such as, e.g., the curious ground state configuration found in a 3D finite size binary Coulomb cluster, ${ }^{5}$ where a mixed arrangement of the two types of particles was observed in the core, while the edge of the system was characterized by shell structures of separated species. Similarly, in 2D systems, we can cite a reentrant melting phenomenon in a $2 \mathrm{D}$ circular confined cluster of repulsive dipoles ${ }^{6,7}$ and interesting asymmetrical ground state configurations observed in symmetrically confined Coulomb clusters. ${ }^{8,9}$

Under specific conditions of density and temperature, an electron gas crystallizes in an ordered structure, forming the so-called Wigner crystal. ${ }^{10}$ In general, a Wigner crystal structure can also refer to an ordered phase occurring in other systems of interacting particles. Several nonelectronic systems show such a Wigner ordered phase, which has been experimentally observed in colloidal systems, ${ }^{11-13}$ dusty plasmas, ${ }^{14-16}$ and ion traps. ${ }^{17,18}$
Depending on the dimensionality and extent of the system, the ordered phase can have a different symmetry. As shown theoretically, the minimum energy arrangement of the 2D infinite electron gas is the triangular lattice, ${ }^{19}$ while the circular confined analog cluster was observed experimentally ${ }^{20}$ and numerically, ${ }^{21}$ and has a mixed ground state configuration with a triangular structure in the core and a circular structure at the edge of the system as a consequence of the symmetry of the external confinement. ${ }^{20,21}$

When the 2D infinite system is subjected to an extra confinement (e.g., parabolic) in one direction, the system can be called quasi-one-dimensional (Q1D). Such a Q1D system of charged particles interacting through a repulsive potential self-organized in a chainlike structure was recently experimentally studied in dusty plasmas ${ }^{15,16}$ and paramagnetic colloids, ${ }^{22,23}$ and through analytical and numerical calculations. ${ }^{24,25}$ In Ref. 24, the authors presented a systematic and interesting study of the structural, dynamical, and thermal properties as a function of the linear density of the system. It is claimed that the one-dimensional (1D) Coulomb crystal confined in a storage ring may be used in atomic clocks or even for quantum computation. ${ }^{26-28}$ An interesting application for the columnar regular structure of interacting particles was shown in Ref. 29, where a regular linear array of superparamagnetic colloidal particles confined in a thin gap was used for the separation of DNA.

Besides the single-component system of interacting particles, which has been widely studied in the past years, Wigner crystallization may also be observed in multicomponent systems of interacting particles. ${ }^{30}$ The simplest multicomponent case is the binary mixture of two types of particles which exhibits a richer set of physical properties when compared to the one-component system. For example, a large number of different equilibrium configurations which are intrinsically dependent on the relative fraction of the different types of particles were recently found in a 2D system of dipoles. ${ }^{31}$ An interesting analysis of the pair correlation function decay as a function of the packing fraction in a 
binary system of hard spheres was presented in Ref. 32. The presence of particles with distinct physical properties (e.g., size, charge, and mass) introduces a competition between different scales, which is the reason for the richer phenomenology in such systems.

In the present paper, we extend the work of Ref. 24 to a binary two-dimensional system of charged particles interacting through a screened Coulomb potential and confined in a parabolic channel. The particles are allowed to move (without friction) in the $x-y$ plane, but due to the external parabolic confinement in the $y$ direction, the system acquires a Q1D character, i.e., the particles are free to move in the $x$ direction and have a constrained motion in the $y$ direction. Due to the possibility of tuning the range of the screened interaction potential in the present model, the binary system of hard spheres ${ }^{32}$ becomes a particular case of our system, which occurs when the screening length of the interaction potential is very small. In spite of the specific interaction potential considered in the present paper, the main qualitative features of the obtained results are expected not to depend on the functional form of the interparticle interaction.

We present a systematic study of the structure of the ground state as a function of the linear density, the ratio between the charges of the distinct types of particles, which characterizes the binary system, and the parameter which determines the range of the repulsive interaction potential between the particles. The range of the interaction between the charged particles can be varied in experimental systems of colloidal particles by changing, for instance, the salt concentration in the solvent. ${ }^{33}$ In the present analysis, we limit ourselves to an equal density of the two types of particles, which are assumed to have the same mass in order to reduce the number of parameters. The normal modes, i.e., the phonons, of the present system are also studied.

The paper is organized as follows. In Sec. II, we describe the model and the procedure used to find the minimum energy configurations. In Sec. III, we study two different zerotemperature phase diagrams. In the first, the ground state configurations are presented for distinct screening parameter of the interaction potential and densities. In the second, the dependence of the crystal structures are shown for different values of the ratio between charges and density. The procedure to find the frequencies of the normal mode spectra and the analysis of the phonon dispersion curves are presented in Sec. IV. Our conclusions are given in Sec. V.

\section{NUMERICAL APPROACH}

We studied a two-dimensional binary cluster consisting of a large and equal number of particles with distinct charges $q_{a}$ and $q_{b}$, which are allowed to move in the $x-y$ plane. The charged particles interact through a repulsive Debye-Hückel (or Yukawa) potential $\exp (-r / \lambda) / r$, are free to move in the $x$ direction, and are confined by a one-dimensional parabolic potential which limits the motion of the particles in the $y$ direction. The potential energy of the system is given by

$$
\begin{aligned}
H= & \sum_{i} \frac{1}{2} m \omega_{0}^{2} y_{i}^{2}+\frac{q_{a} q_{b}}{\epsilon} \sum_{m} \sum_{n} \frac{\exp \left(-\left|\mathbf{r}_{m}-\mathbf{r}_{n}\right| / \lambda\right)}{\left|\mathbf{r}_{m}-\mathbf{r}_{n}\right|} \\
& +\frac{q_{a}^{2}}{\epsilon} \sum_{i>j} \frac{\exp \left(-\left|\mathbf{r}_{i}-\mathbf{r}_{j}\right| / \lambda\right)}{\left|\mathbf{r}_{i}-\mathbf{r}_{j}\right|}+\frac{q_{b}^{2}}{\epsilon} \sum_{k>l} \frac{\exp \left(-\left|\mathbf{r}_{k}-\mathbf{r}_{l}\right|\right) / \lambda}{\left|\mathbf{r}_{k}-\mathbf{r}_{l}\right|},
\end{aligned}
$$

where $\epsilon$ is the dielectric constant of the medium the particles are moving in, $\lambda$ is the Debye length, and $r_{i} \equiv\left|\mathbf{r}_{i}\right|$ is the distance of the $i$ th particle from the center of the confinement potential. In order to reveal the important parameters of the system, it is convenient to write the energy and the distances in units of $E_{0}=\left(m \omega_{0}^{2} q_{a}^{4} / 2 \epsilon^{2}\right)^{1 / 3}$ and $r_{0}=\left(2 q_{b}^{2} / m \epsilon \omega_{0}^{2}\right)^{1 / 3}$, respectively, and to define the quantity $\alpha=q_{b} / q_{a}$ (with $q_{a}=e$ the reference charge taken to be equal to the elementary charge) and the screening parameter $\kappa=r_{0} / \lambda$. In so doing, the expression of the potential energy is reduced to

$$
\begin{aligned}
H= & \sum_{i} y_{i}^{2}+\alpha \sum_{m} \sum_{n} \frac{\exp \left(-\kappa\left|\mathbf{r}_{m}-\mathbf{r}_{n}\right|\right)}{\left|\mathbf{r}_{m}-\mathbf{r}_{n}\right|}+\sum_{i>j} \frac{\exp \left(-\kappa\left|\mathbf{r}_{i}-\mathbf{r}_{j}\right|\right)}{\left|\mathbf{r}_{i}-\mathbf{r}_{j}\right|} \\
& +\alpha^{2} \sum_{k>l} \frac{\exp \left(-\kappa\left|\mathbf{r}_{k}-\mathbf{r}_{l}\right|\right)}{\left|\mathbf{r}_{k}-\mathbf{r}_{l}\right|}
\end{aligned}
$$

and the state of the system is determined now by the ratio between charges $\alpha$, the number of particles (which can be associated with the density), and the dimensionless screening length $\kappa$. The temperature is expressed in units of $T_{0}$ $=E_{0} / k_{B}$, where $k_{B}$ is the Boltzmann constant.

The minimum energy configurations were found, on the one hand, by analytical calculations and, on the other hand, also through molecular dynamics simulations. In the numerical simulations, we typically considered 300 particles, together with periodic boundary conditions in the unconfined direction in order to mimic an infinite system.

The present model system does not address effects due to frictional dissipation. In spite of the primary importance of friction to the motion of the particles in real systems, the ground state configurations are not affected by it.

\section{GROUND STATE CRYSTAL STRUCTURES}

In view of the large parameter space, and in order to show the increased richness of a binary system, we discuss first the case $\alpha=2$. We only considered equal mass particles. If masses are taken different, the two types of particles will feel a different confinement potential which will result in a quantitative modification of the phase diagram. ${ }^{8}$

In this section, we will present the structural results for our binary Q1D system. The results were obtained both analytically and numerically. In the former, we calculate the energy per particle for configurations with different number of chains and different arrangement of the particles within the chains, and minimize such expressions with respect to the distance between chains. To study the dynamical properties of the system, we use the harmonic approximation in order to obtain the normal modes of the ground state configuration. This is shown in Sec. IV.

Before presenting our results, it is convenient to introduce here a dimensionless linear density $(n)$, defined as the ratio 
between the total number of particles in the unit cell of the crystalline arrangement and the length of the unit cell in the unconfined direction. This is similar to the linear density defined previously in Ref. 24.

First, we present the minimum energy configurations and structural transitions as a function of the range of the interaction potential and the linear density of the system. More specifically, we study how the ground state configurations depend on the the screening parameter $(\kappa)$. Next, we study the system as a function of the ratio between the charges of the two types of particles $(\alpha)$. In the present study, we limit ourselves to an equal fraction of particles with distinct charges.

\section{A. Dependence on $\kappa$}

In general, the different charged particles organize themselves in chains. The number of chains, and how particles are placed in them, depends on $n$, the screening parameter $(\kappa)$, and the ratio between the charges $(\alpha)$. In this section, we show how the crystalline structure of the Q1D system depends on $\kappa$ and $n$. The ratio between charges will be kept fixed, $\alpha=2.0$.

Let us first consider the case with $\kappa=1$. When $n$ increases, the following sequence for the first five ground state configurations is observed: one chain $\rightarrow$ two chains $\rightarrow$ four chains (case 1 ) $\rightarrow$ four chains (case 2 ) $\rightarrow$ seven chains (see Fig. 1). The energy per particle as a function of $n$ is shown in Fig. 2(a). As can be seen in Fig. 1, in the one-chain regime, the particles with distinct charges are arranged alternately, and equally spaced on the $x$ axis, where the confinement potential is zero. In this case, the unit cell consists of two particles, one of each type (Fig. 1). The linear density is $n=r_{0} / a$, and the $x$ coordinate of the particles with distinct charges are $x_{i}$ $=2 i a$ and $x_{i}=(2 i-1) a$, with $i= \pm 1, \pm 2, \pm 3, \ldots$. The energy per particle for the one-chain regime is

$$
E_{1}=\left(1+\alpha^{2}\right) \frac{n}{4} \sum_{j=1}^{\infty} \frac{e^{-2 \kappa j / n}}{j}+\alpha \frac{n}{2} \sum_{j=1}^{\infty} \frac{e^{-2 \kappa(j-0.5) / n}}{(j-0.5)},
$$

where the first term accounts for the interaction between particles of the same type, while the last one represents the interaction between particles with different charges.

When $n \approx 0.74155$, the two different particles start to move off the chain, alternating in the positive and negative $y$ directions, leading to a different ground state configuration consisting of two chains. We obtained the following expression for the energy per particle:

$$
\begin{aligned}
E_{2}= & \left(1+\alpha^{2}\right) \frac{n}{4} \sum_{j=1}^{\infty} \frac{e^{-2 \kappa j / n}}{j} \\
& +\alpha \frac{n}{2} \sum_{j=1}^{\infty} \frac{\exp \left[-2 \kappa \sqrt{(j-0.5)^{2}+c^{2}} / n\right]}{\sqrt{(j-0.5)^{2}+c^{2}}}+\frac{c^{2}}{n^{2}},
\end{aligned}
$$

where $c=d / a$ is the dimensionless distance between the chains (Fig. 1). The two-chain regime is characterized by a remarkable asymmetry in the charge distribution. It is interesting to comment that a similar behavior was experimen- tally observed previously in a two-component Coulomb crystal in a linear (quasi-3D) Paul trap and in a binary system of charged dry grains. ${ }^{34,35}$ The charge segregation characterizing the two-chain regime is a consequence of a spontaneous symmetry breaking, which occurs through a continuous structural transition. This is made clear in Fig. 2(b), where the lateral position of the chains is plotted as a function of the density. We found that with the exception of the transition from the one-chain to the two-chain regime, all other structural transitions are characterized by a discontinuity in the lateral position of the chains, which is associated with a first order "structural" transition. In this case, the first derivative of the energy with respect to the density is discontinuous at the transition point [Fig. 2(c)]. For the continuous transition (second order structural changes), a discontinuity is observed only in the second derivative of the energy with respect to the density.

A further increase in the density brings the system to the four-chain regime. In this case, the different types of particles are again symmetrically distributed with respect to the $x$ axis, but still segregated. The four-chain configuration is observed in two different minimum energy configurations, defined here as case 1 and case 2 . In the four-chain (case 1) regime, the rows with the same type of charges are displaced with respect to each other by a distance $a / 4$ along the unconfined direction ( $x$ axis), while neighbor rows with distinct types of particles are displaced by a distance $a / 2$ along the $x$ axis (Fig. 1). Also, the distance between the internal chains (consisting of particles with the same charge) is larger than the distance between the internal chains and the external ones [Fig. 2(b)]. This is interesting because the interaction between chains with distinct charges is intuitively expected to be larger than the interaction between chains with the same lower charge. In the four-chain (case 2) regime, chains with the same charge are displaced by $a / 2$ with respect to each other along the unconfined direction. The distance between chains has an opposite behavior to that of case 1, i.e., the distance between chains with distinct charges is larger than the distance between the internal rows [Fig. 2(b)].

The specific expressions for the energy per particle for the four-chain configurations, as well as for other arrangements presented in Fig. 1, can be obtained directly from Eq. (2). The three-chain configuration shown in Fig. 1 does not become a minimum energy arrangement for $\alpha=2$. However, as will be shown in the next section, for some particular values of $\alpha$, such a configuration was found as the ground state.

As can be observed in Fig. 2, when the density is increased, the system tends to crystallize in structures consisting of a large number of chains. In such cases, it is not trivial to predict the minimum energy arrangement of the particles. To guide our analytical analysis, we resort to numerical molecular dynamic simulations. Due to the larger number of particles considered in the simulations, there are many stable configurations with energy only slightly different from the minimal one. In fact, it is extremely difficult to reach the ground state configuration, even in our numerical simulations. The final configuration typically observed in the simulation was a mixture of several structures. We used this output as a hint to search for the minimal energy arrangement. In Fig. 1, only the stable configurations that have minimal 


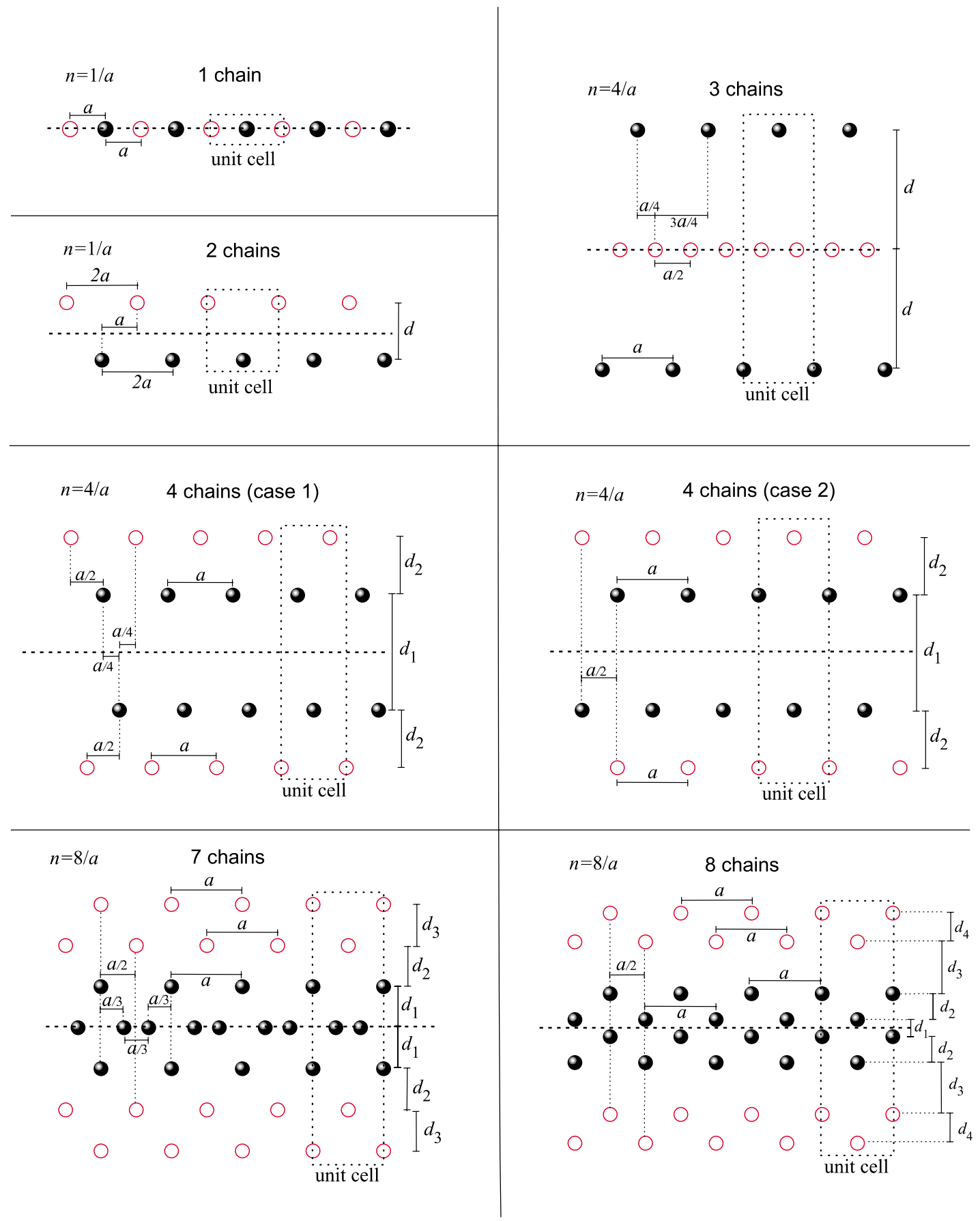

FIG. 1. (Color online) Ground state configurations. The $y=0$ axis is shown by the thin horizontal dotted line, and the two different types of particles in the binary system are indicated by different symbols. The red open circles are particles with charge $q_{b} / q_{a}=\alpha=2$, and the black solid spheres are particles with $q_{a} / e=1$. The linear density of each configuration is also shown.

energy are presented, but many others were considered.

The minimum energy structures presented so far were also found when we minimized the analytical expressions for the energy per particle with respect to the distance between chains for different values of $\kappa$ and $n(\alpha=2)$. The result is presented as a zero-temperature $\kappa-n$ phase diagram in Fig. 3. As a general feature, the interval of density in which a particular phase is observed increases with increasing $\kappa$. Note that the larger the value of $\kappa$, the shorter the range of interparticle interaction potential. This means that the critical dis- tance between adjacent particles necessary to produce an interaction strong enough to change the phase structure will be smaller, and, consequently, the critical density will be larger.

\section{B. Dependence on $\alpha$}

Now we will study how the crystal structure of our system depends on the density $(n)$ when different values of the ratio between charges $(\alpha)$ are considered. In this section, we fixed the screening parameter to $\kappa=1$, which is a typical value in colloidal systems and dusty plasmas. 


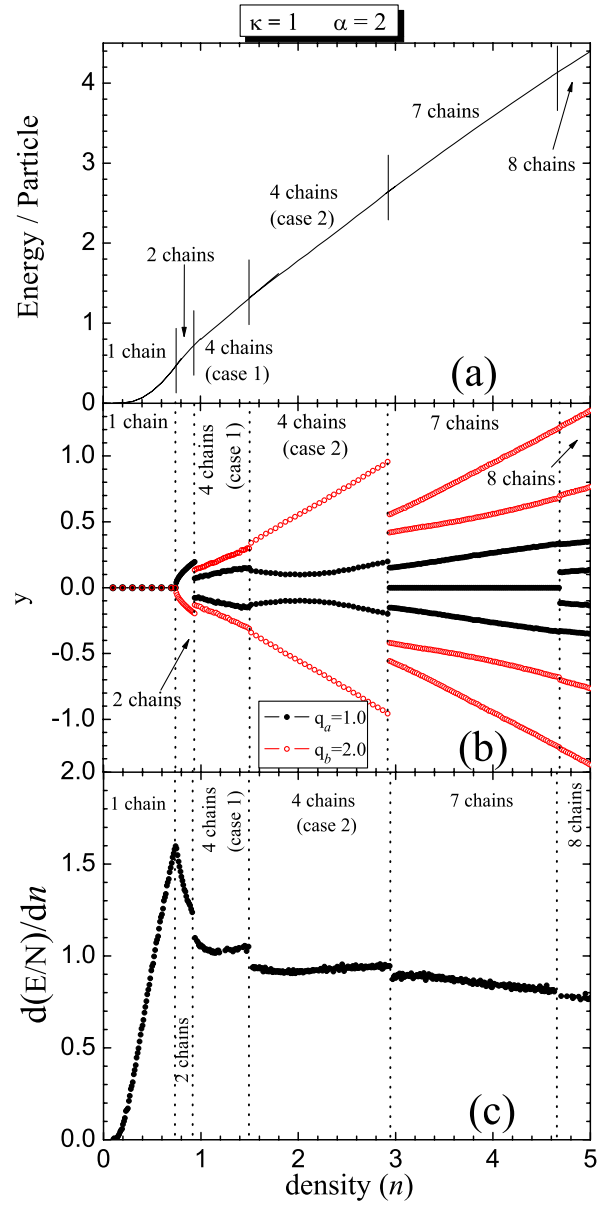

FIG. 2. (Color online) (a) The energy per particle, and (b) the lateral position of the chains as a function of the density for $\kappa=1$ and $\alpha=2$. The black solid circles are the particles with $q_{a} / e=1$ and the red open circles with $q_{b} / e=2$. (c) The first derivative of the energy with respect to the density.

For different values of $\alpha$, the configurations are the same as the ones presented in Fig. 1, but which type of particle is located in the internal chains and in the external chains depends on whether $\alpha$ is $<1$ or $>1$. In general, particles with smaller charge are located in the more internal chains.

The zero-temperature $\alpha-n$ phase diagram is presented in Fig. 4(a). For $\alpha>1$, the same sequence of structures previ-

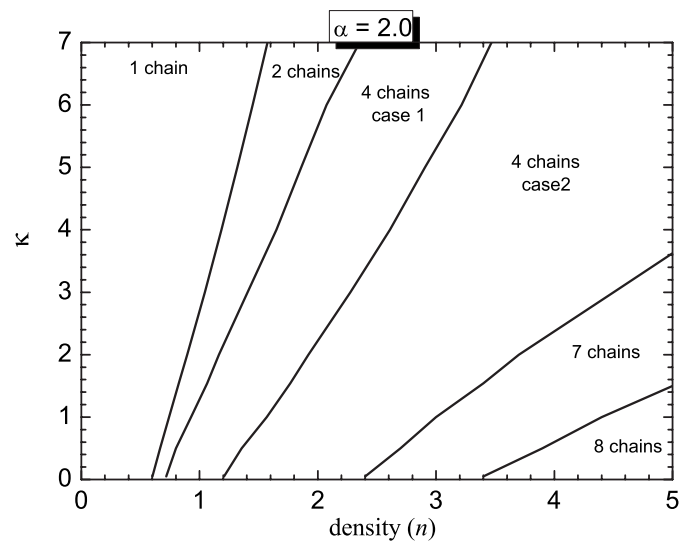

FIG. 3. The zero-temperature $\kappa-n$ phase diagram for $\alpha=2$.
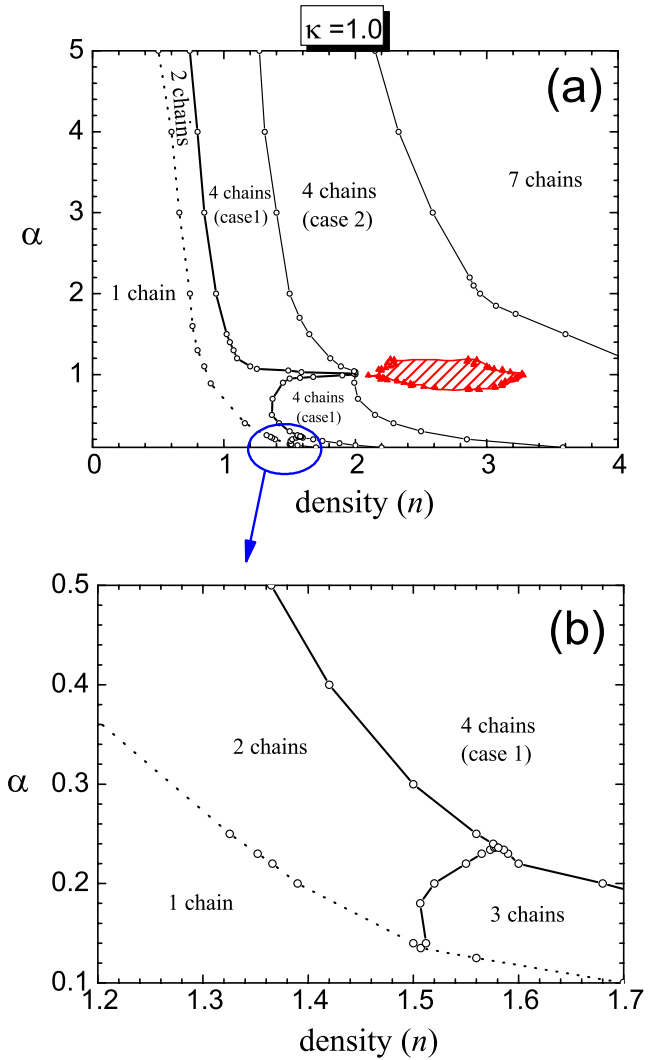

FIG. 4. (Color online) (a) The zero-temperature $\alpha$ - $n$ phase diagram for $\kappa=1$. (b) Zoom of the small $\alpha$ and intermediate density regime. Solid (dotted) lines are first (second) order structural transitions. The symbols are the calculated points. The red dashed region corresponds to the values of $\alpha$ and $n$ for which the two types of particles can be found mixed in the same chain.

ously shown in Fig. 3 is found. The behavior is very different for $\alpha<1$. In this case, new structures appear or disappear depending on the values of $\alpha$ and $n$. For example, an arrangement with three chains (see Fig. 1) is observed for $\alpha$ $\$ 0.237$, as seen in Fig. 4(b). In the interval $0.136 \leqq \alpha$ $\lesssim 0.237$, the three-chain regime appear as the ground state configuration for intermediate density. The two-chain $\rightarrow$ three-chain transition is a first order structural transition.

For $\alpha \lesssim 0.136$, the two-chain regime is no longer observed as the ground state for any value of $n$. Instead, the system changes continuously (second order structural change) from the one- to the three-chain configuration shown in Fig. 1 as the density is increased [Fig. 4(b)] through a zigzag transition. The transition three-chain $\rightarrow$ four-chain transition (case 1 ) is a first order transition for all values of $\alpha$.

For $\alpha=1$, the particles are identical, and the present model is identical to the one studied previously in Ref. 24. We found, in agreement with Ref. 24, that the system presents the following sequence of five different minimum energy structures as the density is increased: one chain $\rightarrow$ two chains $\rightarrow$ four chains $\rightarrow$ three chains $\rightarrow$ four chains $\rightarrow$ five chains. These transitions occur respectively at densities $n$ $=0.8946,2.0312,2.1389,3.3222$, and 4.7054. Notice that such a sequence is different from the one found in the case $\alpha \neq 1$ [Fig. 4(a)]. It should be emphasized that the three- 


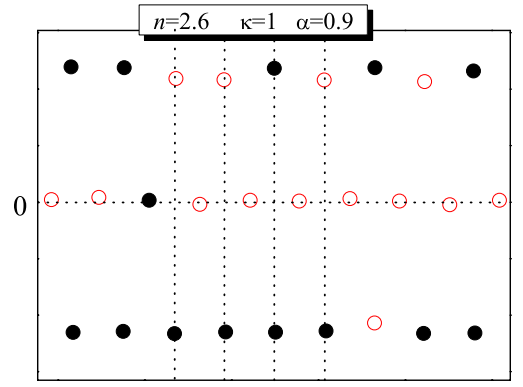

FIG. 5. (Color online) Example of the mixed configuration obtained from molecular dynamic simulations. Black solid circles represent the reference charge $q_{a} / e=1$, while red open symbols are the other particles with charge $q_{b} / e=\alpha$.

chain structure observed when $\alpha=1$ is distinct from the one presented in Fig. 1. In the three-chain configuration of the equal charged particles case, the rows present the same density and are displaced with respect to each other by a distance $a / 2$ along the $x$ axis. This is not the case for the threechain arrangement commented before when $0.136 \lesssim \alpha$ $\lesssim 0.237$ for intermediate values of density (see Fig. 1). In the next section, we directed our attention to the structure of the present binary system when the distinct charges are almost equal $(\alpha \approx 1)$.

\section{Region around $\alpha=1$ : Disordered phase}

Notice that in the density region $1 \leqq n \lesssim 2$, the four-chain (case 1) phase is strongly suppressed in favor of the twochain phase, which for $\alpha=1$ extends now up to $n=2.0312$. Beyond this density, there is a small density interval where the four-chain (case 2) regime is found, which for $\alpha=1$ is identical to the previously found four-chain phase. ${ }^{24}$ In the density interval $2.0312 \lesssim n \lesssim 3.3222$, we found a small region [red shaded area in Fig. 4(a)] where the two types of particles are almost randomly distributed over three chains, which for $\alpha \neq 1$ were found to be no longer perfectly straight (see Fig. 5).

This disordered region, where the chainlike structures are the same as the ones observed in the case $\alpha=1$, is characterized by the presence of particles with distinct charges at the same row. Note that the red dashed region of the $\alpha-n$ phase diagram is inside the region associated with the four-chain (case 2) regime [Fig. 4(a)]. For $n \gtrsim 3.3221$, the four-chain (case 2) regime (Fig. 1) is observed for any value of $\alpha$.

The mixed or disordered configurations with three chains were obtained through molecular dynamic simulations. Only in the mixed region of Fig. 4(a), the energy of such configurations were observed to be smaller than those obtained analytically from the proposed regular structures (Fig. 1). In Fig. 6 , the percentage of the difference in energy between the four-chain (case 2) regime and the mixed configurations inside the mixed region (dashed) of Fig. 4(a) is presented as a function of the density. The largest difference in energy was observed for the case $\alpha=1$, where it was less than $1 \%$.

Note that asymmetric mixed configurations were found recently in a different system of binary charged particles,

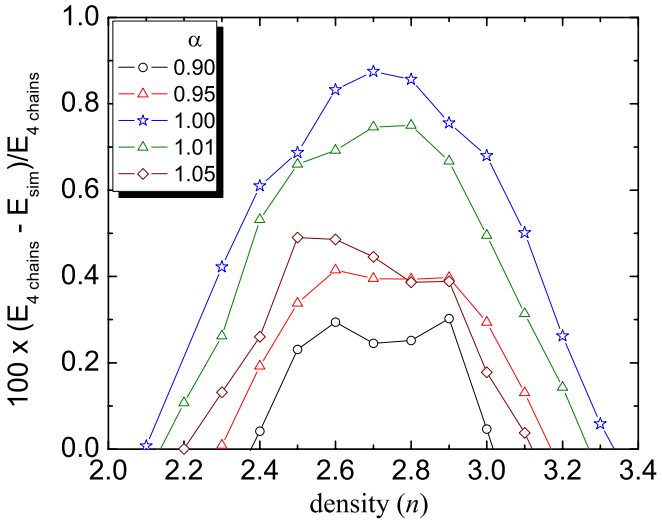

FIG. 6. (Color online) The relative difference between the energy of the four-chain (case 2) regime, obtained analytically, and the energy of the mixed configuration, obtained from molecular dynamic simulations, for values of $\alpha$ in the mixed region presented in Fig. 4(a).

which were confined by a circular parabolic ${ }^{8}$ or hard wall ${ }^{36,37}$ potential.

\section{PHONON SPECTRUM}

We analyze how the normal mode spectrum of the present binary system (in the absence of frictional dissipation ${ }^{38}$ ) behaves as a function of $\alpha, n$, and $\kappa$. Taking into account the translational invariance of the system in the unconfined direction ( $x$ axis), we calculated the normal modes within the harmonic approximation. ${ }^{39}$ Since we are strictly dealing with a two-dimensional system, the number of degrees of freedom per unit cell is twice the number of particles in the unit cell (the unit cell is indicated by dotted boxes in Fig. 1). Therefore, if $l$ is the number of particles per unit cell, there will be $2 l$ branches for the phonon dispersion curves. Half of these branches correspond to oscillations along the chain ( $x$ axis, longitudinal modes), while the others are associated with vi-

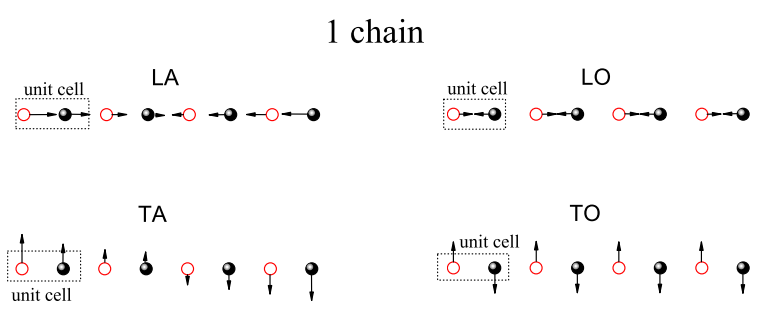

2 chains
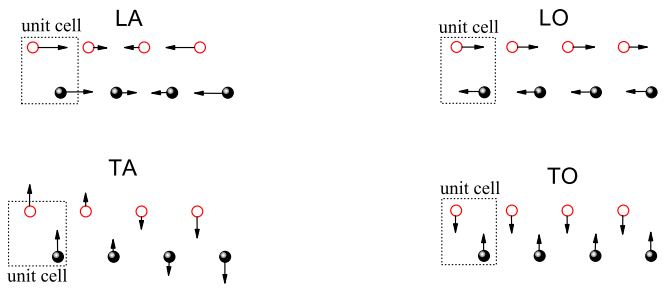

FIG. 7. (Color online) The motion of the particles for each type of normal modes for the one- and two-chain regimes. 

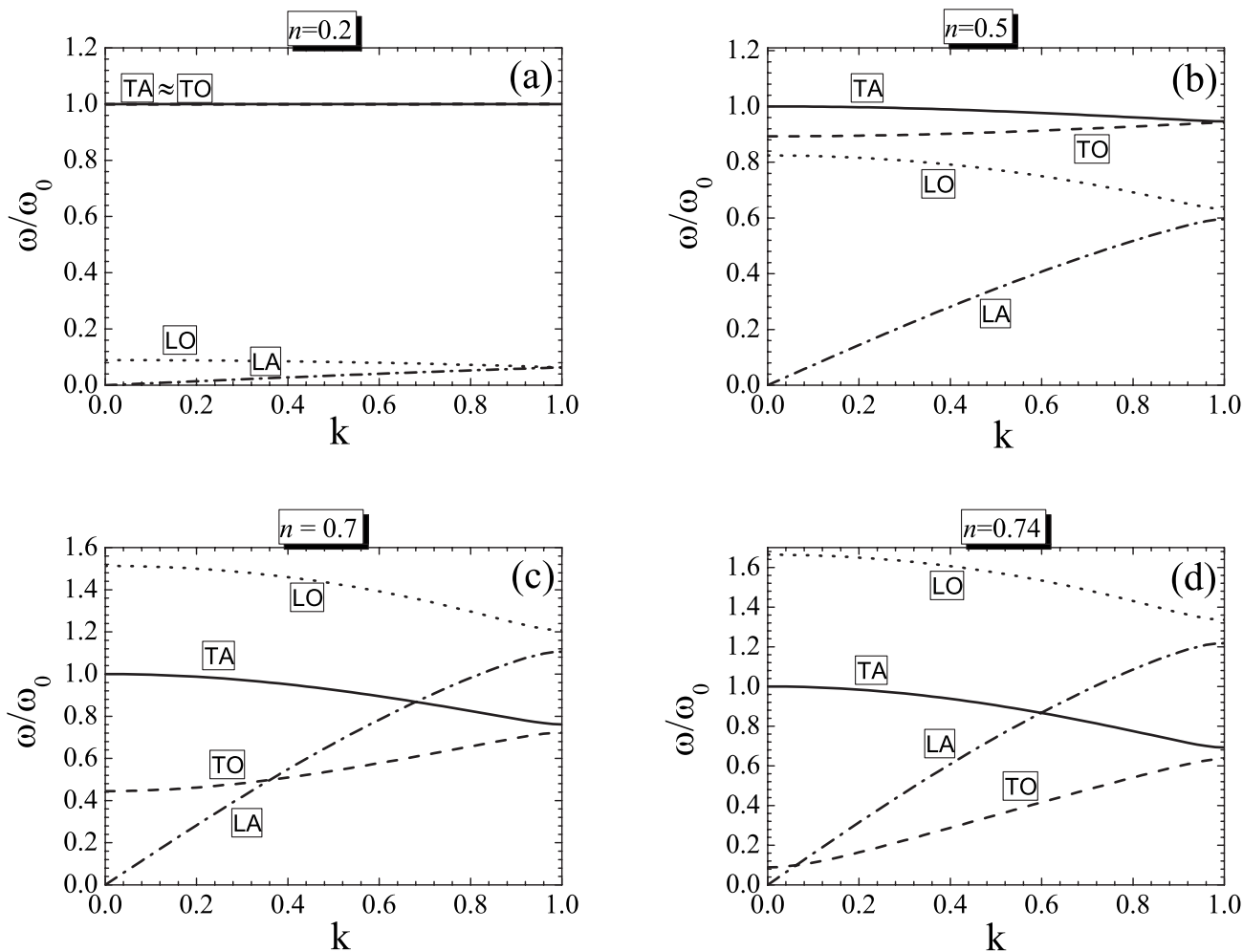

FIG. 8. The phonon spectrum for the one-chain configuration with $\kappa=1$ and $\alpha=2$ for different values of the density. The TA, the TO, the LO, and the LA phonon dispersion curves are identified, respectively, by the solid, dashed, dotted, and dot-dashed curves.

brations along the confinement direction ( $y$ axis, transverse modes). If particles in the unit cell present an in-phase vibration, the mode is also dominantly acoustical, while the opposite out-of-phase oscillation determines the optical mode. In general, a normal mode can be classified in one of the following classes: longitudinal optical (LO), longitudinal acoustical (LA), transverse optical (TO), or transverse acoustical (TA). The motion of the particles for each type of normal mode for the one- and two-chain regimes is illustrated in Fig. 7.

In the harmonic approximation, the normal modes are obtained by solving the system of equations

$$
\left(\omega^{2} \delta_{\mu \nu, i j}-D_{\mu \nu, i j}\right) Q_{\nu, j}=0,
$$

where $Q_{\nu, j}$ is the displacement of particle $j$ from its equilibrium position in the $\nu$ direction, $\mu$ and $\nu$ refer to the spatial coordinates $x$ and $y, \delta_{\mu \nu, i j}$ is the unit matrix, and $D_{\mu \nu, i j}$ is the dynamical matrix, defined by

$$
D_{\mu \nu, i j}=\frac{1}{m} \sum_{u} \phi_{\mu, \nu}(u) e^{-i u q a}
$$

where $u$ is an integer assigned to each unit cell. The force constants are given by

$$
\phi_{\mu, \nu}(u)=\partial_{\mu} \partial_{\nu} \frac{\exp \left[-\kappa \sqrt{\left(x-x^{\prime}\right)^{2}+\left(y-y^{\prime}\right)^{2}}\right]}{\sqrt{\left(x-x^{\prime}\right)^{2}+\left(y-y^{\prime}\right)^{2}}},
$$

with $\left(x-x^{\prime}\right) \in[a u, a(u-1 / 2)]$, and $\left(y-y^{\prime}\right)=$ interchain distance with $(x, y)$ and $\left(x^{\prime}, y^{\prime}\right)$ the equilibrium positions of the particles in the unit cell, and

$$
\phi_{\mu, \nu}(u=0)=-\sum_{u \neq 0} \phi_{\mu, \nu}(u)
$$

The phonon frequency is given in units of $\omega_{0} / \sqrt{2}$. As an example, the complete dynamical matrix for the one- and two-chain regimes is given in the Appendix as an example.

The frequencies for the one- and two-chain configurations are given by

$$
\omega_{l}=\sqrt{\left(A_{1}+A_{3}\right) \pm \sqrt{\left(A_{1}-A_{3}\right)^{2}+4 A_{5}^{2}}}
$$

for the longitudinal modes, and by

$$
\omega_{t}=\sqrt{1+\left(A_{2}+A_{4}\right) \pm \sqrt{\left(A_{2}-A_{4}\right)^{2}+4 A_{6}^{2}}}
$$

for the transverse modes. The parameters $A_{1}, A_{2}, \ldots, A_{6}$ are given in the Appendix. The wave number $k$ for the one- and the two-chain regimes is in units of $\pi / 2 a$, where $2 a$ is the length of the unit cell in the $x$ direction.

In Fig. 8, the phonon spectrum of the system in the onechain regime is presented for different values of the density $n$ and fixed values of $\kappa=1$ and $\alpha=2$. As can be observed, there is a clear dependence of the dispersion curves on $n$. For small values of the density, e.g., $n=0.2$, the transverse modes (optical and acoustical) are almost the same [Fig. 8(a)]. As the density increases, the frequency of the TO mode decreases. An interesting feature to keep in mind is that especially for the TO mode, of the one-chain regime, there is a resulting force in the direction of the confinement potential ( $y$ direction) as a consequence of the repulsive interaction between particles with distinct charges. For a sufficiently small density, the distance between adjacent particles be- 

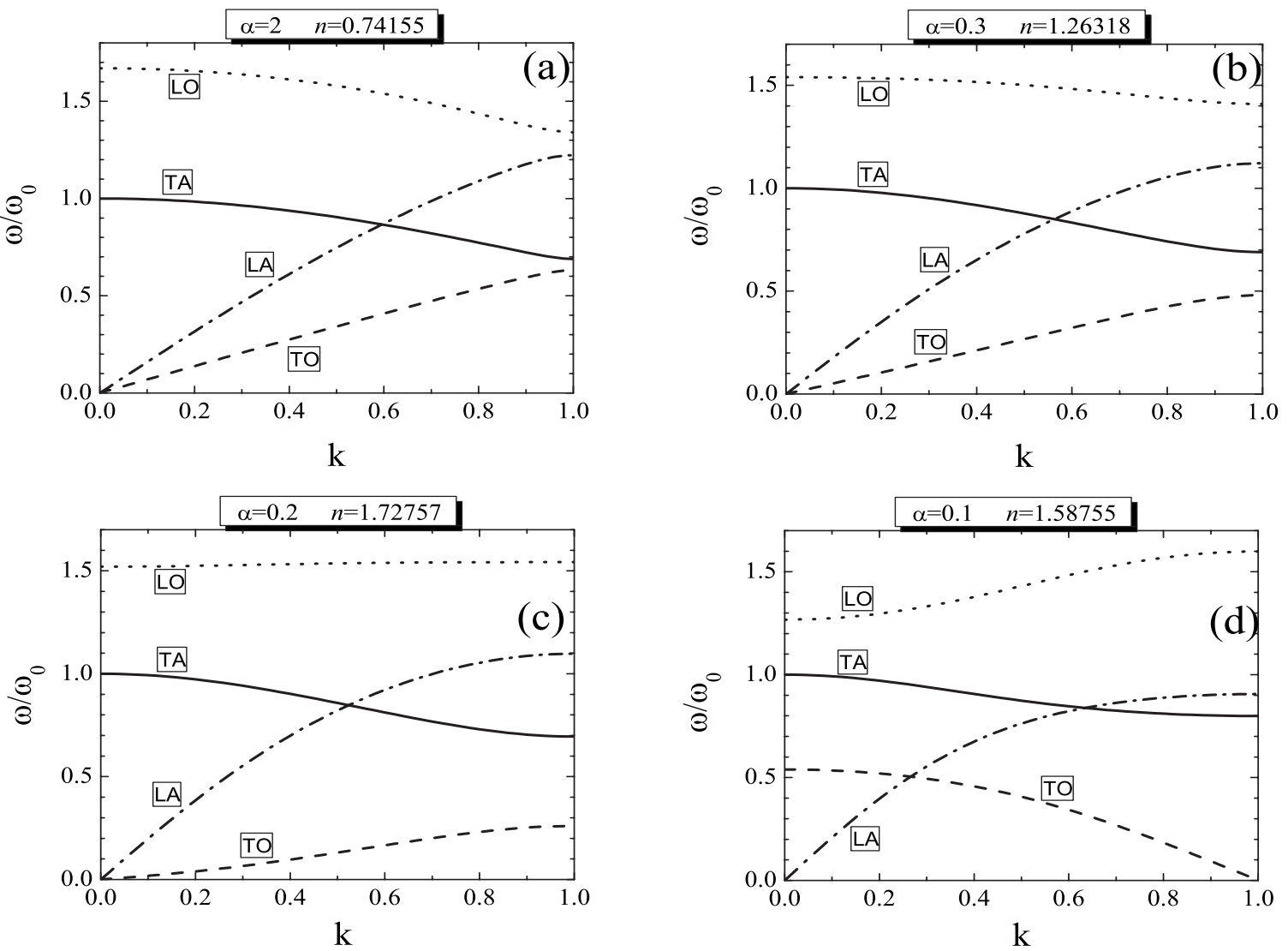

FIG. 9. The normal modes of the system at the transition [(a)-(c)] from the one-chain to the two-chain regime, and (d) from the one-chain to the three-chain structure. The TA, TO, LO, and LA relation dispersion curves are given, respectively, by the solid, dashed, dotted, and dot-dashed curves.

comes large enough to make the interaction between particles irrelevant to affect the oscillation of such particles around the equilibrium positions. In this case, the repulsive force in the $y$ direction is very small and the TO mode will be mainly determined by the confinement potential. A similar explanation can be given for the behavior of the TA mode, but, in this case, the repulsive force in the $y$ direction is smaller than that for the TO mode. Therefore, for a sufficiently small density, the transverse modes will be determined predominantly by the restoring force due to the confinement potential, which is the reason why such modes become almost indistinguishable for small densities.

When the density is increased, the distance between adjacent particles becomes smaller and the repulsive force between them increases and acts as a retarding force. As a consequence, the frequency of the TO oscillations decreases.

The LO mode has the opposite behavior as compared to the TO mode, i.e., there is a hardening of such mode when the density increases. This is a consequence of the larger repulsion due to the closer proximity between particles. A hardening with increasing density is also observed for the LA mode.

For the LA mode, a linear dispersion curve such as $k$ $\rightarrow 0$ is found. This means that in this limit, the longitudinal wave propagates with constant velocity along the chain, and such a velocity increases with increasing density (Fig. 8).

As can be observed in Fig. 8, especially in the limit $k$ $\rightarrow 0$, the TO branch decreases with increasing density. For $n \approx 0.74155$, the system with $\kappa=1$ and $\alpha=2$ undergoes a second order structural phase transition to the two-chain regime. From Fig. 9(a), we notice that the TO mode is zero for $k=0$ and becomes linear in the limit $k \rightarrow 0$, similar to the LA mode. Such a behavior characterizes a continuous structural transition.

In Fig. 9, the dispersion curves for systems with $\kappa=1$ and distinct values of $\alpha$ are presented. In each case, we consider the critical density at which the system transits from the onechain to the two-chain regime [Figs. 9(a)-9(c)] or to the three-chain regime [Fig. 9(d)]. As can be observed in Figs. 9(a)-9(c) for $\alpha=2, \alpha=0.3$, and $\alpha=0.2$, respectively, the TO mode is zero for $k=0$ and is linear for small $k$ values.

In Fig. 9(d), $\alpha=0.1$, the dispersion curve for the TO mode is qualitatively different from the previous ones. The softening of the TO mode is still observed, but now at the edge of the first Brilloum zone $(k=1)$. In this case, the system changes directly from the one-chain to the three-chain regime.

The dependence of the phonon dispersion curves on the linear density for the two-chain configuration, in the case with $\kappa=1$ and $\alpha=2$, is presented in Fig. 10. In general, the frequencies of the longitudinal (transverse) branches increases (decreases) with increasing density. The explanation for such a behavior is similar to the one presented previously for the one-chain regime.

The sound velocity, i.e., $v_{g}=d \omega /\left.d k\right|_{k=0}$, corresponding to the LA phonon mode is shown in Fig. 11 as a function of the 


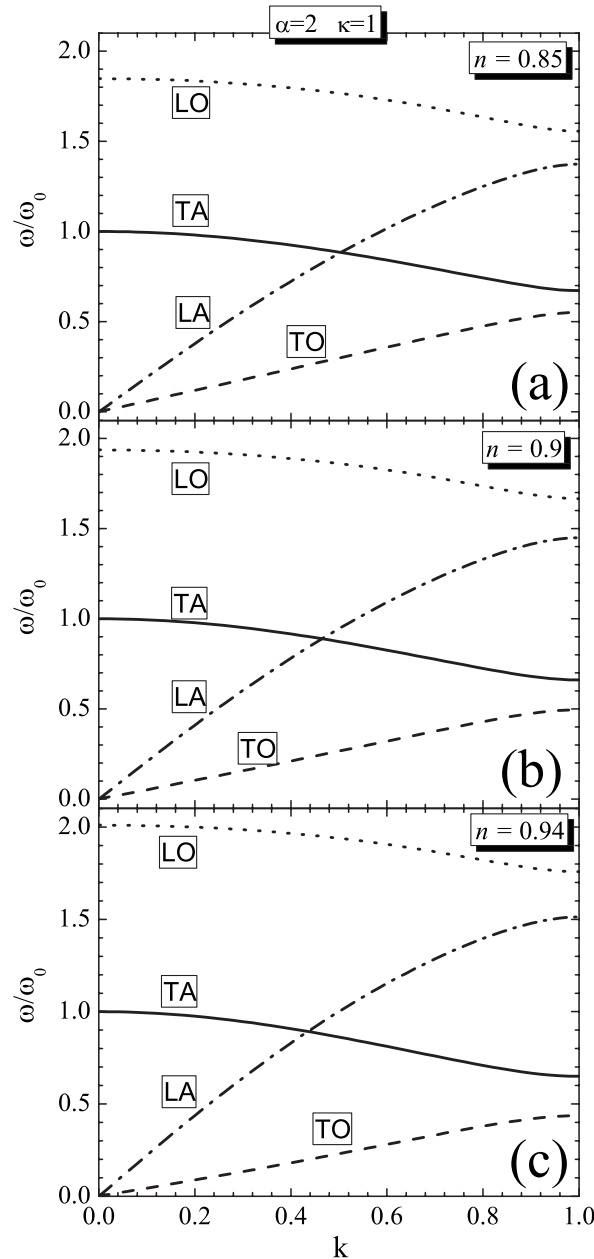

FIG. 10. The phonon spectrum for the two-chain configuration for different values of the density and with $\kappa=1$ and $\alpha=2$. The TA, the TO, the LO, and the LA phonon dispersion curves are identified, respectively, by the solid, dashed, dotted, and dot-dashed curves.

density for $\alpha=2$. Notice that the sound velocity increases with density as expected. At $n=0.74155$, a second order structural phase transition occurs for a two-chain configuration. At this phase transition, the monotonic behavior of $v_{g}$ is changed because of the decrease in the density of particle along each chain and the continuous increase of the separation between the chains.

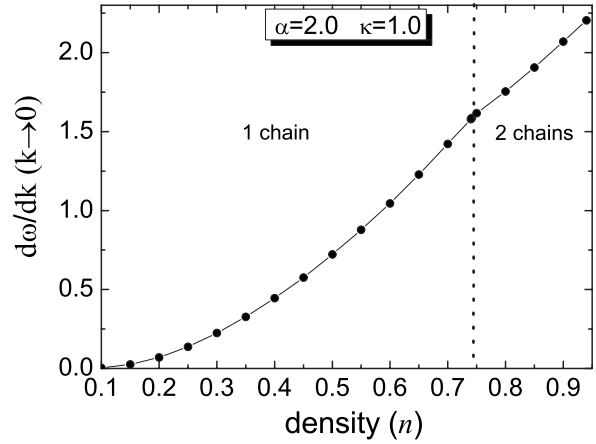

FIG. 11. The sound velocity obtained from the LA phonon mode for the one- and two-chain configurations as a function of density for $\kappa=1$ and $\alpha=2$.
The general qualitative behavior of the phonon dispersion curves presented so far for $\kappa=1$ is also found for different values of the screening parameter.

\section{CONCLUSIONS}

We studied a quasi-one-dimensional binary system of charged particles confined in a parabolic trap. The general structure and the main features of the normal mode spectrum were analyzed. The structural and dynamical properties were studied as a function of the density, the range of the interacting potential, which is an experimental tunable parameter in systems such as colloidal dispersions, dusty plasmas, or even in a binary system of hard spheres, and the parameter characterizing the binary system, namely, the ratio between charges of the two types of particles. We found a very rich variety of ground state configurations, some of them are even not symmetric around the symmetry axis (i.e., $y=0$ ) of the $1 \mathrm{D}$ confinement potential.

The number of chains as well as the internal structure in such chains are a function of the parameters of the system $(\alpha, n$, and $\kappa)$. The set of structures was summarized in zerotemperature phase diagrams which relate the ratio between the distinct types of particles and the screening parameter of the interaction potential with the linear density of the system. The structural transitions between the distinct phases were characterized as being of first or second order. A spontaneous symmetry breaking in the charge distribution of the system was found corresponding to a structural transition that is characterized by a continuous change in the lateral position of the particles. A disordered phase was found in a small region of the four-chain (case 2) part of the phase diagram, where the particles are quasi randomly distributed over three chains. The disordered three-chain configuration transits in a discontinuous way to the four-chain (case 2) ordered phase.

The phonon dispersion curves for the one- and two-chain structures were obtained and analyzed for different values of $\alpha$ and $n$. In general, the normal mode frequencies depend on the linear density of the system. The transverse optical mode decreases monotonically with increasing density, and such a feature was observed for all values of $\kappa$ and $\alpha$. The opposite behavior was observed for the longitudinal modes (optical and acoustical), i.e., there is a hardening of such modes with increasing density.

A remarkable softening of one of the branches of the dispersion curves was found at the second order structural transition. The softening of the TO phonon dispersion curve may occur at the center or at the edge of the first Brillouin zone $(k=\pi / 2 a)$, depending, respectively, on whether the transition is between the one- and the two-chain configuration or between the one- and the three-chain structure.

\section{ACKNOWLEDGMENTS}

Fruitful discussions and collaboration with H. A. Carmona are gratefully acknowledged. W.P.F., P.W.S.O., J.C.N.C., and G.A.F. were supported by the Brazilian National Research Councils: CNPq and CAPES, and the Min- 
istry of Planning (FINEP). F.M.P. was supported by the Flemish Science Foundation (FWO-Vl).

\section{APPENDIX}

The matrix $\omega^{2} \mathbf{I}-\mathbf{D}$ (where $\mathbf{I}$ is the unit matrix and $\mathbf{D}$ is the dynamical matrix) is used in the calculation of the normal modes for the one- and two-chain configurations. The dynamical matrix is of the form

$$
\left[\begin{array}{rrrr}
\omega^{2}-A_{1} & 0 & -A_{5} & 0 \\
0 & \Delta \omega^{2}-A_{2} & 0 & -A_{6} \\
-A_{5} & 0 & \omega^{2}-A_{3} & 0 \\
0 & -A_{6} & 0 & \Delta \omega^{2}-A_{4}
\end{array}\right] \text {, }
$$

where $\Delta \omega^{2}=\omega^{2}-\omega_{0}^{2}$. The quantities $A_{1}$ and $A_{2}\left(A_{3}\right.$ and $\left.A_{4}\right)$ are associated with the interaction between particles with charge $q_{a}\left(q_{b}\right)$, while $A_{5}$ and $A_{6}$ account for the interaction between distinct charges. For the one-chain configuration, such parameters are given by

$$
\begin{aligned}
A_{1}= & \alpha \sum_{j=1}^{\infty} n^{3} \frac{e^{-\kappa(2 j-1) / n}}{(2 j-1)^{3}}\left[2+\frac{2 \kappa(2 j-1)}{n}+\frac{\kappa^{2}(2 j-1)^{2}}{n^{2}}\right] \\
& +\sum_{j=1}^{\infty} n^{3} \frac{e^{-2 \kappa j / n}}{(2 j)^{3}}\left[2+\frac{4 \kappa j}{n}+\frac{(2 \kappa j)^{2}}{n^{2}}\right][1-\cos (2 k j a)] \\
& A_{2}=-\alpha \sum_{j=1}^{\infty} \frac{e^{-\kappa(2 j-1) / n}}{(2 j-1)^{3}}\left[1+\frac{\kappa(2 j-1)}{n}\right] \\
& -\sum_{j=1}^{\infty} n^{3} \frac{e^{-2 \kappa j / n}}{(2 j)^{3}}\left[1+\frac{2 \kappa j}{n}\right][1-\cos (2 k j a)] \\
A_{3}= & \alpha \sum_{j=1}^{\infty} n^{3} \frac{e^{-\kappa(2 j-1) / n}}{(2 j-1)^{3}}\left[2+\frac{2 \kappa(2 j-1)}{n}+\frac{\kappa^{2}(2 j-1)^{2}}{n^{2}}\right] \\
& +a^{2} \sum_{j=1}^{\infty} n^{3} \frac{e^{-2 \kappa j / n}}{(2 j)^{3}}\left[1+\frac{2 \kappa j}{n}\right][1-\cos (2 k j a)] \\
+ & \alpha^{2} \sum_{j=1}^{\infty} n^{3} \frac{e^{-2 \kappa j / n}}{(2 j)^{3}}\left[2+\frac{4 \kappa j}{n}+\frac{(2 \kappa j)^{2}}{n^{2}}\right][1-\cos (2 k j a)] \\
A_{4}= & -\alpha \sum_{j=1}^{\infty} n^{3} \frac{e^{-\kappa(2 j-1) / n}}{(2 j-1}[2 j-1) a \\
&
\end{aligned}
$$

$$
A_{6}=\alpha \sum_{j=1}^{\infty} n^{3} \frac{e^{-\kappa(2 j-1) / n}}{(2 j-1)^{3}}\left[1+\frac{\kappa(2 j-1)}{n}\right]\{\cos [k(2 j-1) a]\} .
$$

The dimensionless wave number $k$ is in units of $\pi / 2 a$.

For the two-chain regime, the parameters in the dynamical matrix are of the form

$$
\begin{aligned}
A_{1}= & \alpha \sum_{j=1}^{\infty} n^{3} \frac{e^{-\kappa r / n}}{r^{3}}\left[(2 j-1)^{2}\left(\frac{3}{r^{2}}+\frac{3 \kappa}{n r}+\frac{\kappa^{2}}{n^{2}}\right)-\left(1+\frac{\kappa r}{n}\right)\right] \\
& +\sum_{j=1}^{\infty} n^{3} \frac{e^{-2 \kappa j / n}}{(2 j)^{3}}\left[2+\frac{4 \kappa j}{n}+\frac{(2 \kappa j)^{2}}{n^{2}}\right][1-\cos (2 k j a)], \\
A_{2}= & \alpha \sum_{j=1}^{\infty} n^{3} \frac{e^{-\kappa r / n}}{r^{3}}\left[\frac{3 c^{2}}{r^{2}}+\frac{\kappa^{2} c^{2}}{n^{2}}+\frac{3 \kappa c^{2}}{n r}-\left(1+\frac{\kappa r}{n}\right)\right] \\
& -\sum_{j=1}^{\infty} n^{3} \frac{e^{-2 \kappa j / n}}{(2 j)^{3}}\left[1+\frac{2 \kappa j}{n}\right][1-\cos (2 k j a)], \\
A_{3}= & \alpha \sum_{j=1}^{\infty} n^{3} \frac{e^{-\kappa r / n}}{r^{3}}\left[(2 j-1)^{2}\left(\frac{3}{r^{2}}+\frac{3 \kappa}{n r}+\frac{\kappa^{2}}{n^{2}}\right)-\left(1+\frac{\kappa r}{n}\right)\right] \\
+ & \alpha^{2} \sum_{j=1}^{\infty} n^{3} \frac{e^{-2 \kappa j / n}}{(2 j)^{3}}\left[2+\frac{4 \kappa j}{n}+\frac{(2 \kappa j)^{2}}{n^{2}}\right][1-\cos (2 k j a)], \\
A_{6}= & -\alpha \sum_{j=1}^{\infty} n^{3} \frac{e^{-\kappa r / n}}{r^{3}}\left[\frac{3 c^{2}}{r^{2}}+\frac{\kappa^{2} c^{2}}{n^{2}}+\frac{3 \kappa c^{2}}{n r}-\left(1+\frac{\kappa r}{n}\right)\right] \\
& \times\{\cos [k(2 j-1) a]\}, \\
& -a_{5}^{2} \sum_{j=1}^{\infty} n^{3} \frac{e^{-2 \kappa j / n}}{(2 j)^{3}}\left[1+\frac{2 \kappa j}{n}\right][1-\cos (2 k j a)], \quad(\mathrm{A} 10) \\
& \times\left\{\sum_{j=1}^{\infty} n^{3} \frac{e^{-\kappa r / n}}{r^{3}}\left[(2 j-1)^{2}\left(\frac{3}{r^{2}}+\frac{3 \kappa}{n r}+\frac{\kappa^{2}}{n^{2}}\right)-\left(1+\frac{\kappa r}{n}\right)\right]\right. \\
A_{4}= & \alpha \sum_{j=1}^{\infty} n^{3} \frac{e^{-\kappa r / n}}{r^{3}}\left[\frac{3 c^{2}}{r^{2}}+\frac{\kappa^{2} c^{2}}{n^{2}}+\frac{3 \kappa c^{2}}{n r}-\left(1+\frac{\kappa r}{n}\right)\right] \\
&
\end{aligned}
$$

where $r=\sqrt{(2 j-1)^{2}+c^{2}}$, and $c=d / a(d$ and $a$ are indicated in Fig. 1) is chosen in order that the eigenvalues of the dynamical matrix be positive. 
*wandemberg@fisica.ufc.br

†rancois.peeters@ua.ac.be

${ }^{1}$ M. E. Leunissen, C. G. Christova, A. P. Hynninen, C. P. Royall, A. I. Campbell, A. Imhof, M. Dijkstra, R. van Roji, and A. van Blaaderen, Nature (London) 437, 235 (2005).

${ }^{2}$ E. V. Shevchenko, D. V. Talapin, N. A. Kotov, S. O'Brien, and C. B. Murray, Nature (London) 439, 55 (2006).

${ }^{3}$ N. Hoffmann, F. Ebert, C. N. Likos, H. Löwen, and G. Maret, Phys. Rev. Lett. 97, 078301 (2006).

${ }^{4}$ A. M. Alsayed, M. F. Islam, J. Zhang, P. J. Collings, and A. D. Yodh, Science 309, 1207 (2005).

${ }^{5}$ T. Matthey, J. P. Hansen, and M. Drewsen, Phys. Rev. Lett. 91, 165001 (2003).

${ }^{6}$ R. Bubeck, C. Bechinger, S. Neser, and P. Leiderer, Phys. Rev. Lett. 82, 3364 (1999).

${ }^{7}$ I. V. Schweigert, V. A. Schweigert, and F. M. Peeters, Phys. Rev. Lett. 84, 4381 (2000).

${ }^{8}$ W. P. Ferreira, F. F. Munarin, K. Nelissen, R. N. Costa Filho, F. M. Peeters, and G. A. Farias, Phys. Rev. E 72, 021406 (2005).

${ }^{9}$ F. F. Munarin, W. P. Ferreira, G. A. Farias, and F. M. Peeters, Phys. Rev. B 76, 035336 (2007).

${ }^{10}$ E. P. Wigner, Phys. Rev. 46, 1002 (1934).

${ }^{11}$ J. Liu, E. M. Lawrence, A. Wu, M. L. Ivey, G. A. Flores, K. Javier, J. Bibette, and J. Richard, Phys. Rev. Lett. 74, 2828 (1995).

${ }^{12}$ K. Zahn, R. Lenke, and G. Maret, Phys. Rev. Lett. 82, 2721 (1999).

${ }^{13}$ M. Golosovsky, Y. Saado, and D. Davidov, Phys. Rev. E 65, 061405 (2002).

${ }^{14}$ J. H. Chu and Lin I, Phys. Rev. Lett. 72, 4009 (1994).

${ }^{15}$ Bin Liu, K. Avinash, and J. Goree, Phys. Rev. Lett. 91, 255003 (2003).

${ }^{16}$ Bin Liu and J. Goree, Phys. Rev. E 71, 046410 (2005).

${ }^{17}$ B. G. Levi, Phys. Today 41(12), 17 (1988).

${ }^{18}$ M. Drewsen, C. Brodersen, L. Hornekær, J. S. Hangst, and J. P. Schiffer, Phys. Rev. Lett. 81, 2878 (1998).

${ }^{19}$ L. Bonsal and A. A. Maradudin, Phys. Rev. B 15, 1959 (1977).
${ }^{20}$ A. Melzer, Phys. Rev. E 67, 016411 (2003).

${ }^{21}$ V. M. Bedanov and F. M. Peeters, Phys. Rev. B 49, 2667 (1994).

${ }^{22}$ Ramin Haghgooie, Chen Li, and Patrick S. Doyle, Langmuir 22, 3601 (2006)

${ }^{23}$ M. Köppl, P. Henseler, A. Erbe, P. Nielaba, and P. Leiderer, Phys. Rev. Lett. 97, 208302 (2006)

${ }^{24}$ G. Piacente, I. V. Schweigert, J. J. Betouras, and F. M. Peeters, Phys. Rev. B 69, 045324 (2004).

${ }^{25}$ Ramin Haghgooie and Patrick S. Doyle, Phys. Rev. E 70, 061408 (2004); 72, 011405 (2005).

${ }^{26}$ G. Birkl, S. Kassner, and H. Walther, Nature (London) 357, 310 (1992).

${ }^{27}$ W. M. Itano and N. F. Ramsey, Sci. Am. 269, 56 (1993).

${ }^{28}$ J. I. Cirac and P. Zoller, Phys. Rev. Lett. 74, 4091 (1995).

${ }^{29}$ Patrick S. Doyle, Jérôme Bibette, Aurélien Bancaud, and JeanLouis Viovy, Science 295, 2237 (2002).

${ }^{30}$ Y. H. Liu, Z. Y. Chen, M. Y. Yu, and A. Bogaerts, Phys. Rev. E 74, 056401 (2006); 73, 047402 (2006).

${ }^{31}$ Lahcen Assoud, René Messina, and Harmut Löwen, Europhys. Lett. 80, 48001 (2007).

${ }^{32}$ Jörg Baumgartl, Roel P. A. Dullens, Marjolein Dijkstra, Roland Roth, and Clemens Bechinger, Phys. Rev. Lett. 98, 198303 (2007).

${ }^{33}$ H. Löwen, J. Phys.: Condens. Matter 13, R415 (2001).

${ }^{34}$ L. Hornekær, N. Kjærgaard, A. M. Thommesen, and M. Drewsen, Phys. Rev. Lett. 86, 1994 (2001).

${ }^{35}$ Amit Mehrotra, Fernando J. Muzzio, and Troy Shinbrot, Phys. Rev. Lett. 99, 058001 (2007).

${ }^{36}$ K. Mangold, J. Birk, P. Leiderer, and C. Bechinger, Phys. Chem. Chem. Phys. 6, 1623 (2004).

${ }^{37}$ K. Nelissen, B. Partoens, I. Schweigert, and F. M. Peeters, Europhys. Lett. 74, 1046 (2006).

${ }^{38}$ G. Piacente, F. M. Peeters, and J. J. Betouras, Phys. Rev. E 70, 036406 (2004).

${ }^{39}$ D. Pines, Elementary Excitation in Solids (Addison-Wesley, New York, 1963), pp. 13 and 20. 